\section{ORIGINAL RESEARCH}

B. Ozgen

K.K. Oguz

A. Cila

\title{
Diffusion MR Imaging Features of Skull Base Osteomyelitis Compared with Skull Base Malignancy
}

BACKGROUND AND PURPOSE: SBO is a life-threatening infection that may have radiologic features similar to those of the neoplastic processes. The purpose of this study was to evaluate the DWI findings in SBO to facilitate the differential diagnosis.

MATERIALS AND METHODS: The MR imaging findings of 9 patients with SBO were retrospectively evaluated and compared with MR imaging studies from 9 patients with NPC, 9 with lymphoma, and 9 with metastatic disease of the skull base. ADC measurements were performed from the $A D C_{S T}$ and the $A D C_{N S T}$ in all 4 groups.

RESULTS: The mean $\mathrm{ADC}_{\mathrm{ST}}$ values were $1.26 \pm 0.19 \times 10^{-3} \mathrm{~mm}^{2} / \mathrm{s}$ for $\mathrm{SBO}, 0.74 \pm 0.18 \times 10^{-3}$ $\mathrm{mm}^{2} / \mathrm{s}$ for NPC, $0.59 \pm 0.11 \times 10^{-3} \mathrm{~mm}^{2} / \mathrm{s}$ for lymphoma, and $0.99 \pm 0.34 \times 10^{-3} \mathrm{~mm}^{2} / \mathrm{s}$ for metastatic disease, respectively. The mean ADC value of SBO was significantly higher than those of NPC and lymphoma $(P<.0001)$. There was no significant difference for the comparison of SBO and metastatic lesions. When an ADC value equal to or higher than $1.08 \times 10^{-3} \mathrm{~mm}^{2} / \mathrm{s}$ was used to rule out lymphoma and NPC, the accuracy was $96 \%$.

CoNCLUSIONS: Although SBO is a relatively rare condition, its differential diagnosis from neoplastic processes of the skull base is essential to start appropriate treatment promptly. ADC values may help to distinguish patients with SBO from those with malignant lesions.

\begin{abstract}
ABBREVIATIONS: $A D C=$ apparent diffusion coefficient; $A D C_{N S T}=A D C$ value of the normalappearing soft tissue; $A D C_{\text {PONS }}=A D C$ value of the central pons; $A D C_{S T}=A D C$ value of the affected soft tissue; DWI = diffusion-weighted imaging; $\mathrm{Dx}=$ diagnosis; $\mathrm{EAC}=$ external auditory canal; Lym = lymphoma; Met = metastatic lesions; NPC = nasopharyngeal carcinoma; $\mathrm{SBO}=$ skull base osteomyelitis; SCC = squamous cell carcinoma; SE = spin-echo
\end{abstract}

$\mathbf{S}^{\text {B }}$ $\mathrm{BO}$ is a severe infection of the skull base that is most frequently seen in elderly patients with diabetes but also in other immunosuppressed patients from all age groups. ${ }^{1-3}$ It is associated with high morbidity and mortality rates despite intensive antibiotic therapy. This condition, which often has a subtle course and nonspecific symptoms such as otalgia and otorrhea, is difficult to diagnose clinically. Similarly, the radiologic findings may be subtle early in the disease and may include signal-intensity changes, enhancement, and an infiltrating pattern indistinguishable from neoplastic processes affecting the skull base such as advanced NPC, lymphoma, and metastatic disease. ${ }^{4-8}$ The purpose of this study was to investigate the DWI findings that would allow prompt and accurate diagnosis of $\mathrm{SBO}$ in a series of patients.

\section{Materials and Methods}

\section{Patients}

A data base search was performed to identify all patients with the clinical and imaging diagnosis of SBO, among patients who had imaging in our institution between March 2003 and February 2010. Nine patients ( 7 men and 2 women) with an age range of 41-81

Received March 9, 2010; accepted after revision June 6.

From the Department of Radiology, Hacettepe University, Ankara, Turkey.

Please address correspondence to Burce Ozgen, MD, Department of Radiology, Hacettepe University, Faculty of Medicine, Sihhiye, Ankara, 06100 Turkey; e-mail: burce@ hacettepe.edu.tr

DOI 10.3174/ajnr.A2237 years (mean, 62 years) were identified. The medical records were also evaluated for pertinent findings. The indications for scanning were as follows: skull base mass (in 3 patients), external otitis (in 4 patients), cerebrovascular accident (in 1 patient), and skull base osteomyelitis in a single patient. In 3 patients, a nasopharynx biopsy was made to rule out malignancy (either before imaging or after an outside scan suggesting malignancy). In 6 patients, biopsy was performed from the external ear canal, middle ear, or mastoid cavity during surgical debridement/mastoidectomy performed either for the identification of the underlying pathogen or for treatment purposes. The diagnosis of SBO was confirmed with histopathologic results in all patients. Laboratory findings also supported the diagnosis of SBO in each case.

A second search, including the same time period, was performed to retrieve cases with the histopathologic diagnosis of stage 4 NPC, skull base lymphoma, and metastatic disease of the skull base. Patients who had their pretreatment MR imaging with diffusion sequences in our institution and the ones who had large tumors centered on the skull base were identified. Among those, the 9 most recent studies were selected, excluding patients younger than 10 years of age and the ones with prior chemotherapy or radiation therapy history. The clinical characteristics of the selected patients were as follows: 7 males and 2 females with an age range of 12-62 years (mean, 44 years) for NPC, 6 males and 3 females with an age range of $14-83$ years (mean, 33 years) for lymphoma, and 8 men and 1 woman with an age range of 46-71 years (mean, 44 years) for metastatic disease. The demographic information of the patients in the 4 groups is summarized in Table 1. Approval of the institutional review board was obtained for this retrospective study. 


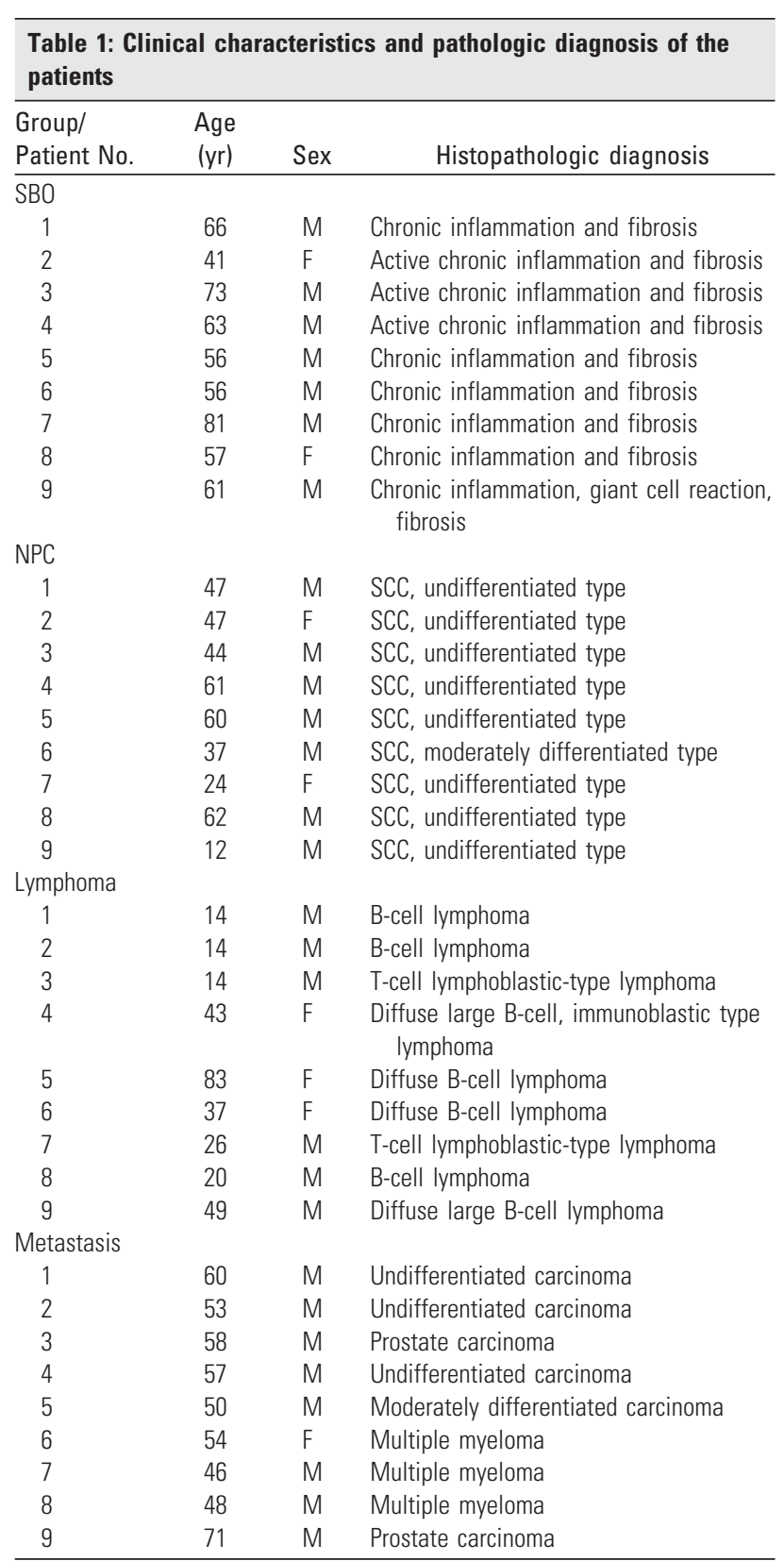

\section{Imaging}

The MR imaging was performed on a 1.5T system (Symphony, Siemens, Erlangen, Germany; or Achieva, Philips Healthcare, Best, the Netherlands). Imaging protocol included axial and sagittal T1weighted SE, axial and coronal T2-weighted turbo SE, and axial and coronal postcontrast T1-weighted SE imaging. All studies were performed as a nasopharynx MR imaging with 3-mm section thickness and fat suppression on T2-weighted and postgadolinium images. Two patients with SBO had no postcontrast T1-weighted imaging (gadolinium could not be administered due to the end-stage renal failure in one and acute renal failure in the other). All studies also included single-shot echo-planar DWI (applied 3 b-values with a maximum of $1000 \mathrm{~s} / \mathrm{mm}^{2}$, TR/TE of 5100/137 ms, matrix of 96-128, and 3-mm section thickness).

\section{Image Evaluation}

A neuroradiologist (B.O.), who was blinded to the cases and was unaware of the histopathologic diagnosis, randomly reviewed the MR images, including the anatomic imaging and the DWI (trace and ADC images).

Although this disease is called "osteomyelitis," the imaging findings in this inflammation are usually much more pronounced in the soft tissues than in the skull base bone marrow. ${ }^{8,9}$ Additionally susceptibility artifacts are much more pronounced within the bone, which can compromise correct ADC measurements. The authors thus decided to obtain ADC measurements from the affected soft tissues beneath the skull base. The $\mathrm{ADC}_{\mathrm{ST}}$ was recorded by manual outlining of a central region of interest of $2 \mathrm{~cm}^{2}$ within the soft-tissue thickening below the skull base (within the posterior parapharyngeal space, anterior to the jugular foramen). The locations of regions of interest were determined with the use of anatomic images. The regions of interest mostly included muscle (the exact content was difficult to evaluate). Same-size $\left(2 \mathrm{~cm}^{2}\right)$ uniform regions of interest were also drawn in the matching normal-appearing soft tissues on the contralateral side to obtain an $\mathrm{ADC}_{\mathrm{NST}}$ for the purposes of normalization and to compare ADC values of normal soft tissues in different subgroups (Fig 1). In patients with bilateral involvement, $\mathrm{ADC}_{\mathrm{NST}}$ measurement was performed from the parapharyngeal space more caudally. The $\mathrm{ADC}_{\mathrm{ST}} / \mathrm{ADC}_{\mathrm{NST}}$ ratios were then calculated for each patient. Because the normal range of ADC values from the brain stem is known from prior studies, an ADC measurement was also obtained from the pons as an internal quality check to assess the validity of our measurements. Calculation of the ADC values was performed by using the software available on workstations provided by the corresponding manufacturer (Leonardo workstations, Siemens; and ViewForum workstations, Philips Healthcare).

The observer making the evaluations noted the presence/absence of image distortion from susceptibility artifacts on the DWI trace images and especially on the ADC maps. None of the cases had artifacts involving the areas where the regions of interest were placed; therefore, the artifacts did not preclude the measurements of the ADC values.

The Wilcoxon signed rank test was used to assess the differences between ADC values of normal and affected soft tissues in patients with SBO. The Kruskal-Wallis test was used for calculating the overall statistical differences among the 4 groups, and the Mann-Whitney $U$ test was performed for calculating the differences in the mean ADC values and the mean $\mathrm{ADC}$ ratios between each pair in the 4 categories.

\section{Results}

The 9 patients with SBO had several comorbidities, including diabetes mellitus in all patients, ischemic heart disease in 3, hypertension in 5, chronic renal failure in 1, and acute renal failure in 1 . All patients had otalgia and otorrhea; 4 patients had accompanying hearing loss. Four patients (44.44\%) also had facial palsy at the time of presentation. The median time from symptom onset to the MR imaging was 6.22 months (with a range of 1-12 months). During this period, at least 6 of the patients had documented oral/topical antibiotic use without clinical improvement. Technetium Tc99m methylene diphosphonate bone scanning was performed in only 1 patient in whom it revealed uptake in the skull base region. The histologic analysis of the biopsy revealed chronic inflammation with granulation tissue and fibrosis (superimposed on an active inflammatory component in 3 patients) (Table 1). The causative agent could not be identified in any patient (most likely due to treatment with antibiotics before diagnostic work-up for SBO), but the SBO was presumed to be bacterial 

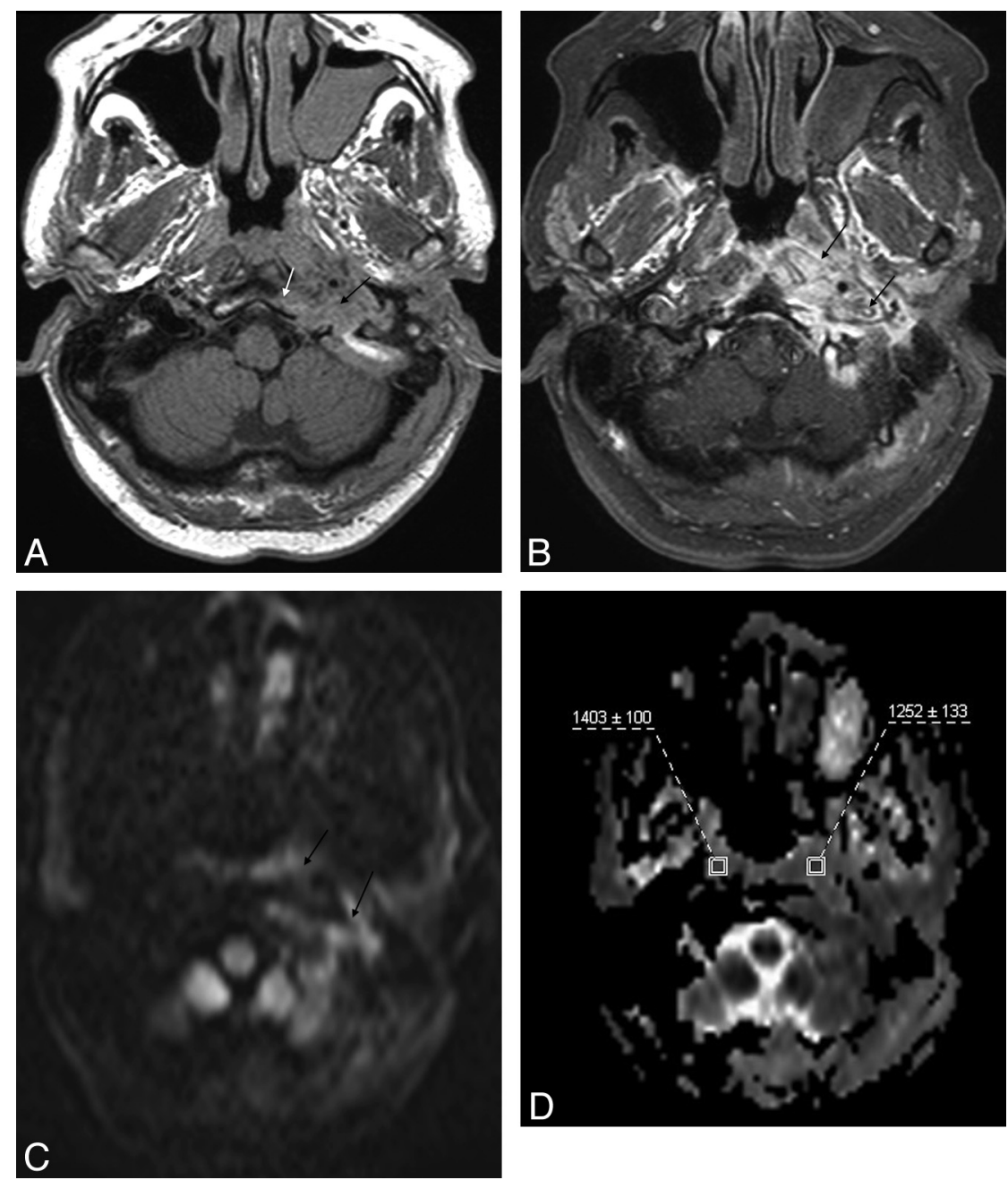

Fig 1. MR images in a case of SBO (patient 8). A, Axial T1-weighted image demonstrates infiltration with decreased T1 signal intensity of the left jugular foramen (black arrow) extending to the poststyloid parapharyngeal space with loss of the cortical margin of the clivus on the left (white arrow). B, Axial postcontrast and fat-suppressed T1-weighted image reveals extensive enhancement of the affected region (arrows). C. Diffusion TRACE image of the same region shows increased signal intensity (arrows). D, Corresponding ADC map with the regions of interest placed to measure the ADC values from the abnormal soft tissues (on the left) and normal-appearing soft tissues (on the right).

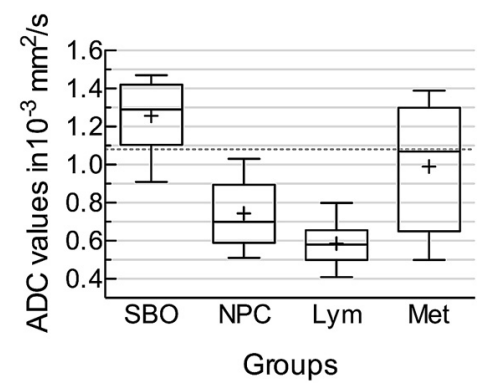

Fig 2. Box-and-whisker plot comparing the mean $A D C$ values of the affected soft tissues in SBO, NPC, lymphoma, and metastatic lesions. The horizontal line is the median (50th percentile) of the measured values; the top and bottom of the box represent the 25th and 75th percentiles, respectively; and whiskers indicate the range from the largest to the smallest observed data points. The plus sign within the whiskers indicates the mean value for each group. Note that despite the overlap between the ADC values of different groups, the ADCs of SBO are significantly higher than those of lymphoma and NPC. The dotted line represents the cutoff value $\left(1.08 \times 10^{-3} \mathrm{~mm}^{2} / \mathrm{s}\right)$ distinguishing SBO from NPC and lymphoma.

in 8 patients and fungal in 1 (according to the patients' responses to antimicrobial treatment). In 5 patients who had completed their antibiotic course with resolution or improvement of their symptoms and clinical findings, normalization of their laboratory results was accepted as successful treatment. Three patients among these had residual cranial neuropathies. One patient was lost to follow-up. Three patients died despite extensive treatment due to multiple organ failure and cardiopulmonary arrest.

The histopathologic diagnosis of patients with NPC was SCC with moderate differentiation in 1 and poor differentiation in 8. Seven patients with lymphoma had B-cell-type lymphoma, and 2 had T-cell lymphoblastic-type lymphoma. Among patients within the metastatic group, 3 had undifferentiated carcinoma, 1 had moderately differentiated carcinoma, 2 had prostate carcinoma, and 3 had multiple myeloma. Although multiple myeloma is a disease characterized by malignant proliferation of the plasma cells and can be classified as a primary bone marrow malignancy, these patients were included in the metastatic group because the imaging is very similar to that in patients with metastatic disease. The histopathologic results of the patients for the 4 groups are also summarized in Table 1.

The mean ADC values for the 4 types of skull base lesions and their statistical analysis is shown in Table 2. In SBO, the mean $\mathrm{ADC}_{\mathrm{ST}}$ value was $1.26 \pm 0.19 \times 10^{-3} \mathrm{~mm}^{2} / \mathrm{s}$ with the 


\begin{tabular}{|c|c|c|c|c|}
\hline Dx & $\mathrm{ADC}_{\mathrm{ST}}$ & $\mathrm{ADC}_{\mathrm{NST}}$ & $\mathrm{ADC}_{\mathrm{ST}} / \mathrm{ADC}_{\mathrm{NST}}$ & $A D C_{\text {PONS }}$ \\
\hline SBO & $1.26 \pm 0.19(0.91-1.45)$ & $1.47 \pm 0.18(1.23-1.80)$ & $0.86 \pm 0.10(0.70-1.02)$ & $0.77 \pm 0.09(0.64-0.89)$ \\
\hline NPC & $0.74 \pm 0.18(0.51-1.03)$ & $1.53 \pm 0.28(1.23-1.9)$ & $0.46 \pm 0.09(0.27-0.57)$ & $0.75 \pm 0.13(0.61-1.05)$ \\
\hline Lymphoma & $0.59 \pm 0.11(0.41-0.80)$ & $1.53 \pm 0.23(1.20-1.87)$ & $0.37 \pm 0.06(0.24-57)$ & $0.79 \pm 0.21(0.67-0.86)$ \\
\hline Metastasis & $0.99 \pm 0.34(0.5-1.39)$ & $1.53 \pm 0.35(1.01-2.12)$ & $0.64 \pm 0.32(0.41-1.38)$ & $0.79 \pm 0.04(0.71-0.85)$ \\
\hline
\end{tabular}

mean $\mathrm{ADC}_{\mathrm{ST}} / \mathrm{ADC}_{\mathrm{NST}}$ ratio of $0.86 \pm 0.10$. For the other groups, the mean $\mathrm{ADC}_{\mathrm{ST}}$ values and the mean $\mathrm{ADC}_{\mathrm{ST}} /$ $\mathrm{ADC}_{\mathrm{NST}}$ ratios calculated were $0.74 \pm 0.18 \times 10^{-3} \mathrm{~mm}^{2} / \mathrm{s}$ and $0.50 \pm 0.13$ for NPC, $0.59 \pm 0.11 \times 10^{-3} \mathrm{~mm}^{2} / \mathrm{s}$ and $0.39 \pm 0.09$ for lymphoma, and $0.99 \pm 0.34 \times 10^{-3} \mathrm{~mm}^{2} / \mathrm{s}$ and $0.68 \pm 0.32$ for metastatic disease, respectively.

The statistical analysis with the Wilcoxon signed rank test demonstrated a significant difference in the ADC values of normal and affected soft tissues $(P=.0078)$ in patients with SBO. There was also a statistically significant difference in the ADC values $(P=.0002)$ and $\mathrm{ADC}$ ratios $(P=.0001)$ of the soft-tissue lesions among the 4 categories by the Kruskal-Wallis test. When the groups were compared with each other with the Mann-Whitney test for independent samples, significant difference was detected among patients with $\mathrm{SBO}$ and NPC as well as among those with SBO and lymphoma $(P<.0001$ for the ADC values and for the ADC ratios). The box-and-whisker plot of the $\mathrm{ADC}_{\mathrm{ST}}$ values of the 4 categories is shown in Fig 2. All patients with lymphoma and all except 1 patient with NPC had ADC values and ADC ratios lower than the lowest values $\left(0.91 \times 10^{-3} \mathrm{~mm}^{2} / \mathrm{s}\right.$ and 0.70 , respectively) observed in patients with $\mathrm{SBO}$. When an $\mathrm{ADC}$ value equal or higher than 1.08 $\times 10^{-3} \mathrm{~mm}^{2} / \mathrm{s}$ and an $\mathrm{ADC}$ ratio $\geq 0.74$ were used to rule out lymphoma and NPC, the accuracy was $96 \%$, with a sensitivity of $89 \%$, specificity of $100 \%$, positive predictive value of $100 \%$, and negative predictive value of $95 \%$.

The ADC value of a single patient with SBO (patient 5 , presumed to have fungal $\mathrm{SBO}$ ) was lower than this $\mathrm{ADC}$ threshold of $1.08 \times 10^{-3} \mathrm{~mm}^{2} / \mathrm{s}$.

There was no statistically significant difference between the $\mathrm{ADC}_{\mathrm{ST}}$ values and $\mathrm{ADC}$ ratios of patients with $\mathrm{SBO}$ and patients with skull base metastases $(P=.0770)$. When the cutoff ADC value of $1.08 \times 10^{-3} \mathrm{~mm}^{2} / \mathrm{s}$ was used to differentiate patients with SBO from all 3 groups of patients with neoplastic lesions of the skull base, the accuracy was $86 \%$, with a sensitivity of $89 \%$, specificity of $85 \%$, positive predictive value of $67 \%$, and negative predictive value of $96 \%$.

The mean $\mathrm{ADC}_{\mathrm{NST}}$ and $\mathrm{ADC}_{\mathrm{PONS}}$ values were calculated and are demonstrated in Table 2. When these mean $\mathrm{ADC}_{\mathrm{NST}}$ and $\mathrm{ADC}_{\mathrm{PONS}}$ values were compared, no significant difference was found among the 4 categories $\left(P=.9694\right.$ for $\mathrm{ADC}_{\mathrm{NST}}$ and $P=.4579$ for $\left.\mathrm{ADC}_{\mathrm{PONS}}\right)$, confirming the validity of the measurements.

\section{Discussion}

$\mathrm{SBO}$ is an uncommon aggressive infection, which can be challenging to diagnose and treat. ${ }^{2}$ In most patients, it arises from the spread of an ear infection called "malignant or necrotizing otitis externa." The process originates as a soft-tissue infection of the EAC, then spreads to the skull base through the fissures of Santorini (along the floor of cartilaginous EAC) and then to the tympanomastoid suture and along the fas- cial planes. ${ }^{2,3,10,11}$ It causes bone destruction of the skull base and involves the skull base foramina, causing cranial neuropathies. This condition is typically seen in an elderly patient with diabetes as illustrated by our patients who all had diabetes. ${ }^{2,3}$ The affected individuals usually present with otalgia, otorrhea, and hearing loss, as seen in our patients. Although the previously reported mortality rate of $50 \%$ has decreased to $0 \%-15 \%$, this disorder is still difficult to cure. ${ }^{12,13}$ In our group of patients, the mortality and morbidity rates were $33.3 \%$ and $60 \%$, respectively. It is, therefore, crucial to make the correct diagnosis to prevent progression of the inflammation to the skull base foramina and to prevent fatal complications.

Although in the setting of appropriate clinical and laboratory findings, the combination of technetium and gallium scintigraphies can suggest the diagnosis, patients presenting with cranial neuropathies often undergo radiologic evaluation with CT or MR imaging, which could be nonspecific. ${ }^{14-16}$ The typical radiologic changes in SBO include hypointensity of the clival bone marrow signal intensity on T1-weighted images, infiltration and obliteration of the parapharyngeal fat planes, decreased T1 signal intensity, and enhancement within the soft tissues beneath the skull base and infiltration of the retrocondylar fat (as illustrated in Fig 3). ${ }^{5,8,9,17}$ However, for the inexperienced reader, the appearance of the soft tissues (especially on T1-weighted images) and accompanying bone marrow changes may suggest a skull base malignancy such as advanced NPC, metastatic disease, or lymphoma. ${ }^{5-8} \mathrm{We}$, therefore, sought to evaluate the value of DWI as a possible technique to assist in the differential diagnosis.

DWI has been investigated for its capability to characterize tissue in various diseases and was found to increase the accuracy of imaging in distinguishing malignant and benign masses as well as discriminating metastatic nodes from benign lymphadenopathies. ${ }^{18-23}$ The diffusion characteristics of the tissue depend on its microstructure (its cellularity, nucleus/ cytoplasm ratio, nature of the extracellular matrix, and so forth) and its perfusion (micromotion of molecules in its capillaries). ${ }^{24}$ In general, malignant neoplasms with enlarged nuclei, hypercellularity, and a reduced extracellular matrix have reduced $\mathrm{ADC}$ values, whereas benign neoplasms have higher $\mathrm{ADC}$ values. ${ }^{25}$ Our results also reflect these changes- that is, the mean $\mathrm{ADC}_{\mathrm{ST}}$ values for $\mathrm{SBO}$ were higher than those of neoplastic tissue groups. The difference was significant for NPC and for lymphoma, the major differential diagnoses in the patients with SBO. ${ }^{5} \mathrm{ADC}$ values of all except 1 patient with SBO were higher than the ADC values of patients with NPC and lymphoma. The exception was the patient with SBO who improved after the antifungal treatment. This finding is indeed consistent with previously published reports showing decreased ADC values in some fungal abscesses. ${ }^{26,27}$

Normally inflammation causes an increase in the extracel- 

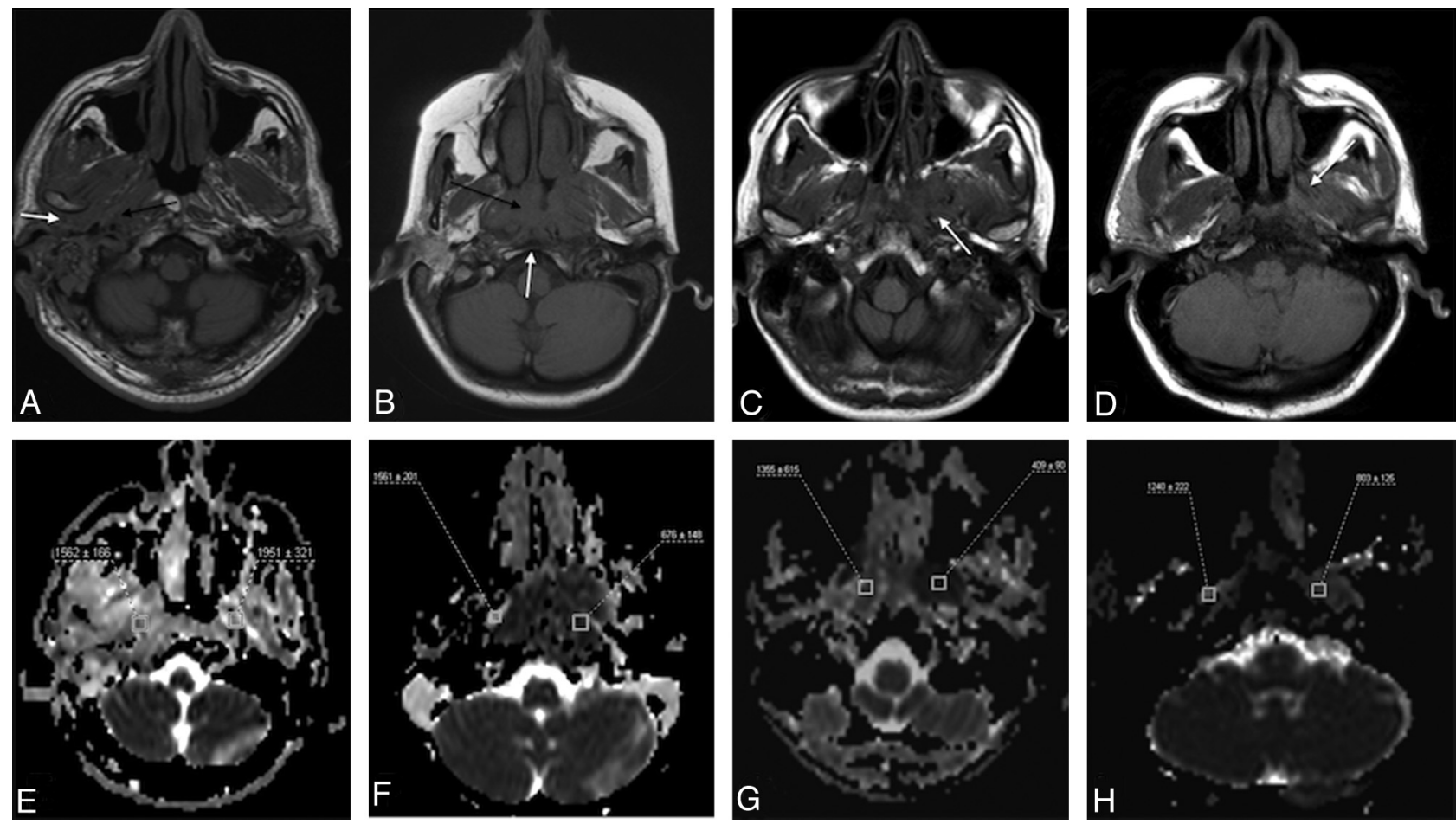

Fig 3. MR images (anatomic images and $A D C$ maps) of patients from each group. $A$, Axial T1-weighted image of a patient with SBO demonstrates infiltration of the soft tissues around the $\mathrm{EAC}$, in the retrocondylar fat space (white arrow), extending to the poststyloid parapharyngeal space and jugular foramen (black arrow). $B$, Axial T1-weighted image of a patient with NPC reveals a large infiltrative mass in the nasopharynx (black arrow) with a small area of clival infiltration (white arrow). C, Axial T1-weighted image of a patient with lymphoma with a low T1-signal-intensity mass around the left pterygoid process (arrow), extending to the masticator space. D, Axial T1-weighted image of a patient with undifferentiated carcinoma metastatic to the skull base with the low T1-signal-intensity metastases on the left (arrow). $E-H$, Corresponding ADC maps of the patients with SBO, NPC, lymphoma, and metastases, respectively, with the regions of interest placed to measure the ADC values.

lular water, resulting in an increase of the ADC values. ${ }^{28}$ In our study, although the $\mathrm{ADC}_{\mathrm{ST}}$ values of the affected soft tissues in $\mathrm{SBO}$ were higher than those in the neoplastic lesions, the values were lower than those in $\mathrm{ADC}_{\mathrm{NST}}$. The mean $\mathrm{ADC}_{\mathrm{ST}}$ value in $\mathrm{SBO}$ was also lower than previously reported values $(2.76 \pm$ $0.32 \times 10^{-3} \mathrm{~mm}^{2} / \mathrm{s}$ ) of ADC in the inflammatory tissues in the head and neck. ${ }^{28}$ This result may, of course, be related to the characteristics of the magnet and may also be affected by the small size of our study group. However, the mean $\mathrm{ADC}_{\mathrm{NST}}$ $\left(1.47 \pm 0.18 \times 10^{-3} \mathrm{~mm}^{2} / \mathrm{s}\right)$ obtained for comparison was within the average values reported for normal ADC of the soft tissues $\left(1.488-1.608 \times 10^{-3} \mathrm{~mm}^{2} / \mathrm{s}\right) .^{29}$

In a study evaluating the CT and MR imaging appearances of necrotizing otitis externa, the authors reported relatively low T2 signal intensity of the abnormal soft tissues beneath the skull base and concluded that the histopathologic characteristics of the disease with a denser matrix and associated fibrosis would explain the relative decrease of T2 signal intensity. ${ }^{17}$ Indeed the histopathologic evaluation of the presented patients with SBO revealed fibrosis accompanying chronic inflammation in all patients, which may also explain our observation of a relative decrease of ADC values in SBO relative to the normal soft tissue of the skull base.

Apart from benign/malignant discrimination of the lesions, DWI may further demonstrate specific diffusion parameters related to the histopathologic characteristics of the lesions. It has been shown that the ADC values are significantly lower in the neck masses and lymphadenopathies of lymphoma than in SCC. ${ }^{18-20}$ Correspondingly in our study, the mean ADC value of patients with lymphoma was lower than that of patients with NPC. The mean ADC value for lymphoma was similar to the values reported in the literature $\left(0.65 \pm 0.09\right.$ to $\left.0.66 \pm 0.17 \times 10^{-3} \mathrm{~mm}^{2} / \mathrm{s}\right) .{ }^{18-20}$ However, in our study, the mean ADC value of SCC of the nasopharynx was lower than that previously reported $(0.96 \pm 0.11$ to $1.13 \pm$ $\left.0.43 \times 10^{-3} \mathrm{~mm}^{2} / \mathrm{s}\right) .{ }^{19,20}$ This might have resulted from the fact that $8 / 9$ of our patients with NPC had NPC of an undifferentiated type (Table 1), and the ADC values of poorly differentiated carcinomas are known to be lower than those in differentiated tumors. ${ }^{18}$

The ADC values of metastatic lesions showed a great variability $\left(0.5-1.39 \times 10^{-3} \mathrm{~mm}^{2} / \mathrm{s}\right)$, resulting in a large overlap with lesions of patients with SBO and NPC. The mean ADC value of metastatic lesions was significantly different only from the mean ADC of lymphoma. This variability (as seen in Fig 2) may be partly due to the histologically heterogeneous aspect of the metastatic group (because our case numbers were very limited, it was not possible to subgroup those lesions into homogeneous groups) but is probably mostly related to the primary histologic characteristics of the metastatic lesions, some having high ADC values. However, it would be interesting to evaluate the DWI findings of multiple myeloma in comparison with SBO, lymphoma, and NPC in further studies.

There were several limitations to our study. The small number of patients is a major limitation, and though most of our statistical results were significant and consistent with the literature, studies with larger series are needed to validate the above-described results. Although SBO is an infection of the bone, we preferred to make our measurements from the soft tissues and not from the bone. This decision was made for 
several reasons: First, it is difficult to outline a region of interest, and there are usually susceptibility artifacts in the skull base marrow. Also the skull base bony involvement was subtle in a few patients with SBO and lymphoma. Additionally, the measured diffusion sequences were performed on 2 different MR imaging scanners, which might possibly affect the measured ADC values, but the results from different scanners are being routinely used as comparisons in the literature. Besides, comparable results of normal soft tissue of the skull base and the pons obtained from each scanner support our belief that the use of various magnets is not a major limitation, but more investigation is needed in this field to make a definite assessment.

\section{Conclusions}

SBO, a potentially life-threatening disorder, is frequently evaluated by MR imaging, but the lack of specific findings may hinder the differential diagnosis from neoplastic processes affecting the skull base, causing delay in the diagnosis and treatment. Our study demonstrated that DWI might help in this setting, especially to distinguish SBO from lymphoma and NPC, which may appear similar on conventional sequences. However, further experience and studies with larger groups are needed to make a definite statement on this subject.

\section{References}

1. Rubin J, Yu VL. Malignant external otitis: insights into pathogenesis, clinical manifestations, diagnosis, and therapy. Am J Med 1988;85:391-98

2. Sreepada GS, Kwartler JA. Skull base osteomyelitis secondary to malignant otitis externa. Curr Opin Otolaryngol Head Neck Surg 2003;11:316-23

3. Carfrae MJ, Kesser BW. Malignant otitis externa. Otolaryngol Clin North Am 2008;41:537-49, viii-ix

4. Slattery WH 3rd, Brackmann DE. Skull base osteomyelitis: malignant external otitis. Otolaryngol Clin North Am 1996;29:795-806

5. Chang PC, Fischbein NJ, Holliday RA. Central skull base osteomyelitis in patients without otitis externa: imaging findings. AJNR Am J Neuroradiol 2003;24:1310-16

6. Clark MP, Pretorius PM, Byren I, et al. Central or atypical skull base osteomyelitis: diagnosis and treatment. Skull Base 2009;19:247-54

7. Subburaman N, Chaurasia MK. Skull base osteomyelitis interpreted as malignancy. J Laryngol Otol 1999;113:775-78

8. Karantanas AH, Karantzas G, Katsiva V, et al. CT and MRI in malignant external otitis: a report of four cases. Comput Med Imaging Graph 2003;27:27-34

9. Kwon $\mathrm{BJ}, \mathrm{Han} \mathrm{MH}, \mathrm{Oh} \mathrm{SH}$, et al. MRI findings and spreading patterns of necrotizing external otitis: is a poor outcome predictable? Clin Radiol 2006;61:495-504

10. Nadol JB Jr. Histopathology of pseudomonas osteomyelitis of the temporal bone starting as malignant external otitis. Am J Otolaryngol 1980;1:359-71

11. Bernheim J, Sade J. Histopathology of the soft parts in $\mathbf{5 0}$ patients with malignant external otitis. J Laryngol Otol 1989;103:366-68

12. Chandler JR. Malignant external otitis. Laryngoscope 1968;78:1257-94

13. Narozny W, Kuczkowski J, Mikaszewski B. Infectious skull base osteomyelitis: still a life-threatening disease. Otol Neurotol 2006;27:1047-48, author reply 1048

14. Parisier SC, Lucente FE, Som PM, et al. Nuclear scanning in necrotizing progressive "malignant" external otitis. Laryngoscope 1982;92:1016-19

15. Uri N, Gips S, Front A, et al. Quantitative bone and 67Ga scintigraphy in the differentiation of necrotizing external otitis from severe external otitis. Arch Otolaryngol Head Neck Surg 1991;117:623-26

16. Seabold JE, Simonson TM, Weber PC, et al. Cranial osteomyelitis: diagnosis and follow-up with In-111 white blood cell and Tc-99m methylene diphosphonate bone SPECT, CT, and MR imaging. Radiology 1995;196:779-88

17. Grandis JR, Curtin HD, Yu VL. Necrotizing (malignant) external otitis: prospective comparison of CT and MR imaging in diagnosis and follow-up. $R a$ diology 1995;196:499-504

18. King AD, Ahuja AT, Yeung DK, et al. Malignant cervical lymphadenopathy: diagnostic accuracy of diffusion-weighted MR imaging. Radiology 2007; 245:806-13

19. Maeda M, Kato H, Sakuma H, et al. Usefulness of the apparent diffusion coefficient in line scan diffusion-weighted imaging for distinguishing between squamous cell carcinomas and malignant lymphomas of the head and neck. AJNR Am J Neuroradiol 2005;26:1186-92

20. Wang J, Takashima S, Takayama F, et al. Head and neck lesions: characterization with diffusion-weighted echo-planar MR imaging. Radiology 2001;220:621-30

21. Abdel Razek A, Soliman N, Elkhamary S, et al. Role of diffusion-weighted MR imaging in cervical lymphadenopathy. Eur Radiol 2006;16:1468-77. Epub 2006 Mar 24

22. Razek AA, Sadek AG, Kombar OR, et al. Role of apparent diffusion coefficient values in differentiation between malignant and benign solitary thyroid nodules. AJNR Am J Neuroradiol 2008;29:563-68. Epub 2007 Nov 26

23. Srinivasan A, Dvorak R, Perni K, et al. Differentiation of benign and malignant pathology in the head and neck using $3 \mathrm{~T}$ apparent diffusion coefficient values: early experience. AJNR Am J Neuroradiol 2008;29:40-44

24. Le Bihan D, Breton E, Lallemand D, et al. Separation of diffusion and perfusion in intravoxel incoherent motion MR imaging. Radiology 1988;168:497-505

25. Guo AC, Cummings TJ, Dash RC, et al. Lymphomas and high-grade astrocytomas: comparison of water diffusibility and histologic characteristics. Radiology 2002;224:177-83

26. Gaviani P, Schwartz RB, Hedley-Whyte ET, et al. Diffusion-weighted imaging of fungal cerebral infection. AJNR Am J Neuroradiol 2005;26:1115-21

27. Charlot M, Pialat J-B, Obadia N, et al. Diffusion-weighted imaging in brain aspergillosis. Eur J Neurol 2007;14:912-16

28. Kito S, Morimoto Y, Tanaka T, et al. Utility of diffusion-weighted images using fast asymmetric spin-echo sequences for detection of abscess formation in the head and neck region. Oral Surg Oral Med Oral Pathol Oral Radiol Endod 2006; 101:231-38. Epub 2005 Dec 27

29. Srinivasan A, Dvorak R, Rohrer S, et al. Initial experience of 3-Tesla apparent diffusion coefficient values in characterizing squamous cell carcinomas of the head and neck. Acta Radiol 2008;49:1079-84 